\title{
Making connections for a biopsychosocial future in cancer care
}

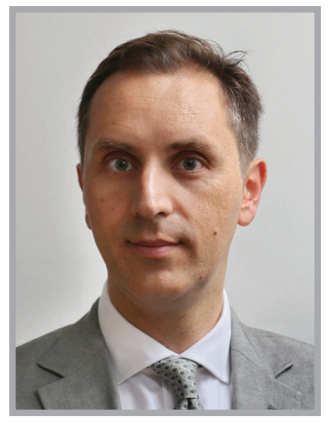

Alex King*
"We hope that this special issue will stimulate us to learn, practice and explore together, and keep the social, psychological and biomedical facets of cancer connected."
First draft submitted: 15 October 2016; Accepted for publication: 17 October 2016; Published online: 10 November 2016

This special issue of Future Oncology marks a unique opportunity for connecting physical and psychosocial aspects in cancer research and clinical practice.

In the context of the last decades' increasingly effective and decreasingly toxic biomedical treatments, as well as vivid social advocacy against stigma, attention to patient experience, quality of life, psychosocial adaptation and mental health broadly, the agenda of 'survivorship' or 'living with, and beyond cancer' - is no longer an afterthought $[1,2]$.

Yet, specialties may be making progress in parallel, rather than jointly and collaboratively. For instance, new trials of targeted treatments offering the promise of increased survival for advanced melanoma are proceeding apace, yet the high rates of associated distress have received little systematic consideration [3]. We cannot just assume that connections, however obvious, will just happen, without the opportunities to come together.

I often like to surprise my medical colleagues with the striking finding that 'the survival benefit associated with marriage was larger than the published survival benefit of chemotherapy' [4]. In this review of cancer treatment studies, drawing on data from over 730,000 people in the USA, married patients (especially men) were less likely to present with metastatic disease, more likely to received active treatment and less like to die of cancer - and this effect was stronger than the effects of chemotherapy treatments per se. After pondering the practicalities of prescribing marriage, we get further into a conversation about the deeper connections and synergies between the social, psychological and medical processes involved in getting people into, through and recovering from cancer treatment. We can talk about cancer as a profound psychological experience of threat, loss and change, and how warmth, trust and connection are as powerful and necessary as much as our best technologies.

This special issue is a similarly striking moment, a forum offering a vivid opportunity for a connected discussion. We can now bring concepts, evidence and solutions from psycho-oncology to the broader oncology audience, and develop opportunities to sustain connections at multiple levels.

\section{KEYWORDS}

- biopsychosocial • cancer

- integrative $\bullet$ multidisciplinary

- oncology • psycho-oncology 
Some contributions in this issue focus on the value of connections between people with cancer and the significant people in their lives. For instance, Pini and colleagues [5] explored the experiences of teenagers and young adults with cancer in relation to their education and school peer groups in the UK. Using the vivid method of using photos taken by the young people themselves as prompts for in-depth qualitative interviews, they brought to the surface themes such as the value of remaining connected and 'being thought about' at school, as well as the social tension inherent in wishing to be seen as 'just normal' yet equally 'pulling out the cancer card'. These insights offer clinical and educational services a direct way to include the voices of young people in service improvement. At other side of the age spectrum, Goldzweig and colleagues [6] study the complex dyadic connections between hope and distress within Israeli couples of oldestold people ( $>85$ years) with cancer. The interplay between patient and carer distress does vary with age, and this study focuses sensitively on an understudied segment: physically well people of advanced age, in active cancer treatment. Among other findings, it is surprising to see high distress and low hope among patients being contrasted with the exact opposite in carers. Both these studies caution us against facile assumptions about 'average' patients and 'typical' needs, and toward thoroughly personalized support possibly, a dimension where psychosocial care is by necessity more advanced than medicine.

Some contributions focus on the connections between clinical specialties necessary to provide truly state-of-the-art holistic care for patients and carers. Sagar [7] describes how multidisciplinary psychoeducation programs can be weaved in and around medical treatment pathways, to develop patient activation and self-management. Such embedded programs (including decision support, prehabilitation, peer support and rehabilitation) use psychological insights and technologies to the benefit of every patient within the clinical pathway. Yet for the (sizeable) minority with heightened and complex distress, relatively small proportions will get through from screening to accepting direct intervention [8], and it is timely to consider how we develop and offer options that 'invisibly' weave psychological care into routine medical care. At another level of connection, Antoni and colleagues [9] review the evidence and potential for psychological interventions to influence biophysical parameters. Specifically, interventions targeting stress in post-surgical breast cancer patients can show promising results in short-term psychosocial, neuroendocrine and immunological function, as well as potential for long-term survival benefits. The sophisticated cross-disciplinary expertise required to explore this potential will surely serve to build valuable bridges between specialties.

Bringing it all together, it is an honor to welcome the commentary by Watson and Dunn [10]. With deep experience, international perspective and a vivid vision for psycho-oncology, they write concisely and convincingly of the value in fully integrating psycho-oncology into cancer care, from prevention onward. They call for "a willingness to see beyond the immediate pressures to deal with cancer as a biological disease" and take the necessary extra steps to develop the procedures, relationships and connections between specialties. We hope that this special issue will stimulate us to learn, practice and explore together, and keep the social, psychological and biomedical facets of cancer connected.

\section{Financial \& competing interests disclosure}

The author has no relevant affliations or financial involvement with any organization or entity with a financial interest in or financial conflict with the subject matter or materials discussed in the manuscript. This includes employment, consultancies, honoraria, stock ownership or options, expert testimony, grants or patents received or pending, or royalties.

No writing assistance was utilized in the production of this manuscript.

\section{References}

1 Jacobsen PB, Holland JC, Steensma DP. Caring for the whole patient: the science of psychosocial care. J. Clin. Oncol. 30(11), 1151-1153 (2012).

2 Walsh K. Addressing psychosocial issues in cancer survivorship: past, present and future. Future Oncol. 12(24), 2823-2834 (2016).

3 Dunn J, Watson M, Aitken JF, Hyde MK. Systematic review of psychosocial outcomes for patients with advanced melanoma. Psychooncology doi:10.1002/pon.4290 (2016) (Epub ahead of print).
4 Aizer AA, Chen M-H, McCarthy EP et al. Marital status and survival in patients with cancer. J. Clin. Oncol. 31(31), 3869-3876 (2013).

5 Pini S, Gardner P, Hugh-Jones S. How teenagers continue school after a diagnosis of cancer: experiences of young people and 
recommendations for practice. Future Oncol. 12(24), 2785-2800 (2016).

6

Goldzweig G, Baider L, Andritsch E,

Rottenberg Y. Hope and social support in elderly patients with cancer and their partners: an actor-partner interdependence model. Future Oncol. 12(24), 2801-2809 (2016).
7 Sagar SM. Integrative oncology: are we doing enough to integrate psycho-education? Future Oncol. 12(24), 2779-2783 (2016).

8 Funk R, Cisneros C, Williams RC, Kendall J, Hamann HA. What happens after distress screening? Patterns of supportive care service utilization among oncology patients identified through a systematic screening protocol. Support. Care Cancer 24(7), 2861-2868 (2016).
9 Amiel CR, Fisher HM, Carver CS, Antoni $\mathrm{MH}$. The importance of stress management among postresection breast cancer patients. Future Oncol. 12(24), 2771-2774 (2016).

10 Watson M, Dunn J. The multidisciplinary art and science of cancer care: integrating psycho-oncology. Future Oncol. 12(24), 2775-2778 (2016). 